\title{
Ultrastructural analysis application for spermatozoa study in male infertility
}

\author{
Pichugova Svetlana $V^{1,2 *}$, Tulakina Lyudmila G', Klein Alexey $V^{1}$ and Beykin Yakov $B^{1,2}$ \\ ${ }^{1}$ Clinical and Diagnostic Centre, Ekaterinburg, Russian Federation \\ ${ }^{2}$ Institute of Immunology and Physiology of the Ural Branch of the Russian Academy of Sciences, Ekaterinburg, Russian Federation
}

\begin{abstract}
Against the background of the unfavorable trend of recent years to intensify the role of male factor in infertile marriages the problem of male reproductive health gained not only medical but also social importance. Nowadays male infertility is the cause of reproductive problems of about $30-50 \%$ of couples. Therefore, research work in male infertility diagnostics and treatment is relevant and has high priority [1]. Male infertility is defined as a consequence of pathological processes in the body that contribute to the development of pathospermia. Factors that cause male fertility disorder and lead to infertility are thoroughly studied and well characterized. Factors that because male infertility include: varicocele [2-5], changes in biochemical parameters of ejaculate [6,7], infectious and inflammatory diseases of the urogenital system [8-11], immune infertility [12,13], endocrine disorders [14-17], drug addiction [18], smoking and alcohol addiction [20-22]. Some of them are involved in reproductive function regulation (hormones, cytokines, proteins, carbohydrates, lipids). The negative impact of such factors on spermatogenesis will be shown as its levels deviation from the accepted reference interval. Other factors and conditions have direct damaging effects on the reproductive system (infectious agents, antisperm antibodies, unfavourable environmental conditions, smoking, alcohol, drugs, varicocele).
\end{abstract}

\section{Introduction}

Despite the diversity of the causes of male reproductive function disorders, ejaculate analysis is the main among all methods of laboratory and instrumental studies used to determine the functional state of gonads and male fertility. Ejaculate analysis became more informative and it gives important clinical information about spermatogenesis and functional competence of spermatozoa [23]. The most frequent pathological evidence in the ejaculate is asthenozoospermiaspermatozoa motility disorder that can be a significant cause of male reproductive function disorder $[24,25]$. Such disorders may have genetic nature, as well as being the result of external factors. The question of what internal spermatozoa changes happen at the ultrastructural level in spermatozoa motility disorder remains not enough studied.

The purpose of the study was to examine spermatozoa pathological changes at the ultrastructural level in infertility, which could cause its motility disorder.

\section{Materials and methods}

For the purpose of the study ejaculates, obtained after three days of abstinence, we mechanically collected from 100 men aged 25-42 years old that were examined for infertility. Materials were taken using blinding method and at the same time were sent to routine examination (spermogram) and electron microscopic spermatozoa study.

Spermogram was carried out using conventional methods and its parameters were evaluated according to World Health Organization criteria 2006 [23]. Spermatozoa motility was evaluated in the following categories:

\footnotetext{
"A" category - fast progressive movement;

"B" category - slow progressive movement;
}

"C" category - all types of no progressive movement;

"D" category - immovable spermatozoa.

The reference group consisted of samples in which, according to light microscopy, spermatozoa motility corresponded to standard criteria: at least $25 \%$ of "A" category or $50 \%$ of "A" + "B" categories.

For the purpose of an electron microscopic spermatozoa study ejaculate was fixed in $2.5 \%$ glutaraldehyde solution followed by $1 \%$ osmium dioxide solution re-fixation. The sample was then dehydrated in alcohol in increasing concentration and polymerized at a temperature of 60 degrees Celsius. Ultrathin sections were taken using Leica EM UC6 ultra microtome, contrasted with lead citrate and examined in Morgagni 268 electron microscope.

In total, more than 2000 sections from 100 men were examined and 1560 microphotographs taken at $\mathrm{x} 4400$ to $\mathrm{x} 71000$ magnifications.

\section{Study results}

From 3450 spermograms carried out by "Clinical and Diagnostic Centre" over the period of 2012-2014, the structure of the obtained results shows that the majority of spermogram parameters changes falls on spermatozoa motility disorder (Figure 1).

As long as spermatozoa motility directly depends on the state of

Correspondence to: Pichugova Svetlana V, Clinical and Diagnostic Centre, 620144, Ekaterinburg, 8 Marta street, 78 “B”, Russian Federation, Russia, Tel: +79058021539; E-mail: ekb-lem@mail.ru

Key words: infertility, reproduction, pathology

Received: September 19, 2016; Accepted: October 04, 2016; Published: October 06, 2016 


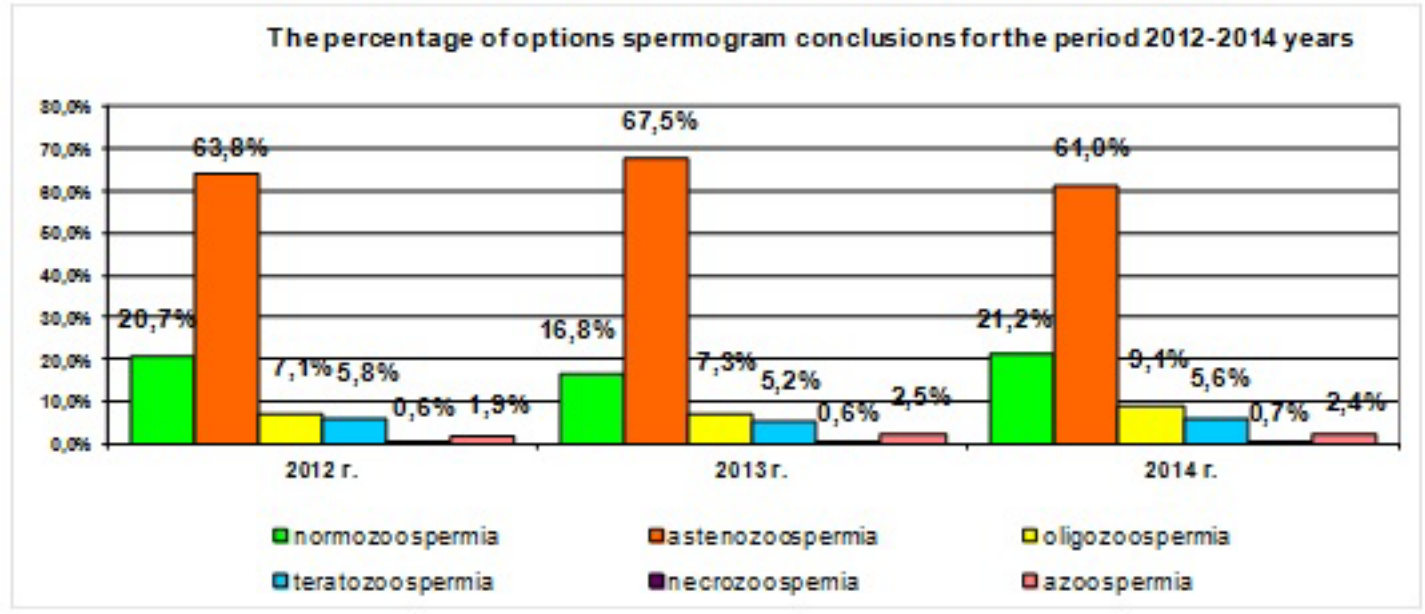

Figure 1. Spermogram versions, carried out by "Clinic and Diagnostic Centre" over the period of 2012-2014.

spermatic filament, filament ultrastructure was precisely studied in the first place.

Patients of the reference group (30 patients) were diagnosed normozoospermia and according to the results of an electron microscopic study mitochondria of filament middle part had clear double-contoured membranes, cristas and had spiral boxing (Figure 2a). Filament axoneme in cross sections consisted of nine peripherals and one central pairs of duplets, radial spokes (Figure 2b), fibrous layer was formed by ordered fibrils. In addition to the filaments the structure of spermatozoa heads and necks was also evaluated. Spermatozoa heads had mature homogenous chromatin, clear straight acrosome, which occupied the larger area of the head (Figure 2c). Neck structures were clearly visible: basal plate, centriole, segmented columns (Figure 2d).

Speaking about patients with asthenozoospermia (70 patients) at the ultrastructural level in spermatozoa filament it was found that in all cases axoneme contained all ultrastructural components and had typical structure (Figure 2b). In the filament middle part in all cases mitochondria were swollen to different extent, blurring, destruction of cristas and clarification of mitochondrial matrix were present, in most cases mitochondria spiral boxing was broken (Figures 3a and 3b). There were identified spermatozoa with twisted filament enclosed in cytoplasmic droplet, in which hyperplastic nuclear membrane was seen, that is considered to be a variant of spermatozoa dysplasia (Figure 3c). There was an increase of spermatozoa quantity that had displayed vacuolated or granular chromatin in its heads or combination of these pathological changes (Figure 3d). There were also diagnosed changes in spermatozoa heads acrosome: swelling, vacuolization, premature degradation (Figures 3d, 3e, 3f). Spermatozoa neck and axoneme structures remained intact (Figure 2d).

\section{Results consideration}

Asthenozoospermia is one of the most common spermatozoa abnormal changes. Spermatozoa motility disorders occurred when pathological changes affected mitochondria, or when mitochondria pathological changes were combined with nuclear membrane hyperplasia. Generally, mitochondria's swelling was identified. Mitochondria pathological changes also include membranes doublecontour disorder, cristas destruction, matrix homogenization, in severe cases - mitochondria clarification and devastation, mitochondria shift relative to each other. The data obtained shows that mitochondria are
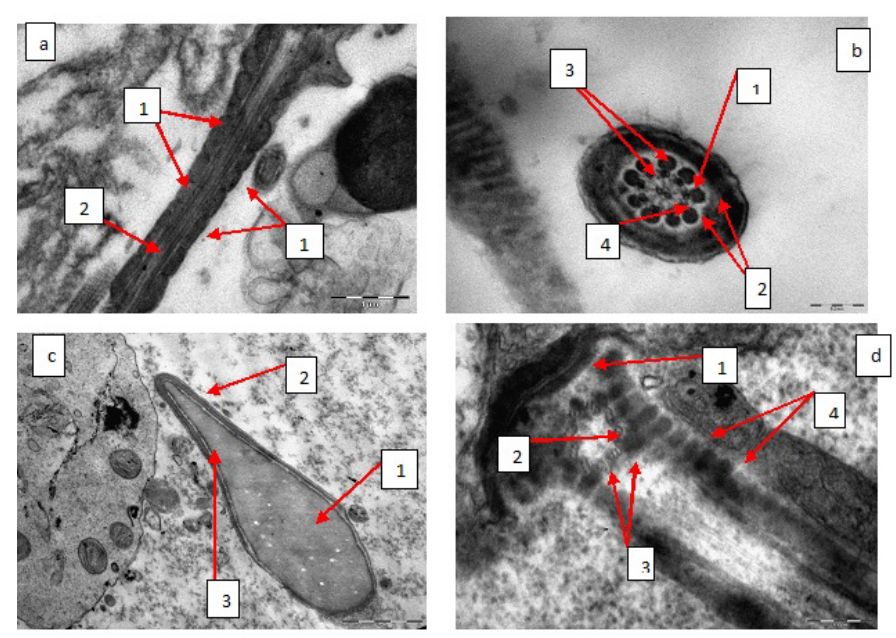

Figure 2. a - spermatozoa filament middle part, mitochondria typical structure (1), spiral boxing around axoneme (2), x 14 000; b - filament cross section at the middle part level, axoneme typical structure, central pair of duplets (1), peripheral duplets (2), additional tight fibrils (3), dynein knobs (4), x 56 000; c-spermatozoa head, typical structure, mature homogenous chromatin (1), acrosome (2), fissured postacrosomal area (3). x 14000 ; d-spermatozoa neck, typical structure, basal plate (1), centriole (2), centriole triplets (3), segmented columns (4), x 71000 .

the most vulnerable and susceptible to pathological changes organelles, other ultrastructures are considerably stable. It may be explained by a number of features. Mitochondria are the only organelles that have autonomous DNA [26], and any structural change can break mitochondrial respiratory function that is necessary in the early stages of spermatogenesis for biosynthesis, and in mature spermatozoa for its movement, and this structural change may cause spermatozoa motility lowering [27,28]. Another vulnerable factor of mitochondria is its localization. In filaments mitochondria in a form of a turbinal chain are located in the middle part for the purpose of quick adenosine triphosphoric acid transfer to axomene and in order to provide spermatozoa motility. Mitochondria swelling, its shifting or wrong positioning in spiral boxing breaks power transfer mechanism for spermatozoa movement, which primarily results in different stages of asthenozoospermia [28-30].

The origin of such ultrastructural deficiency as hyperplastic nuclear membrane has little explanation in literature. It may be possible that 

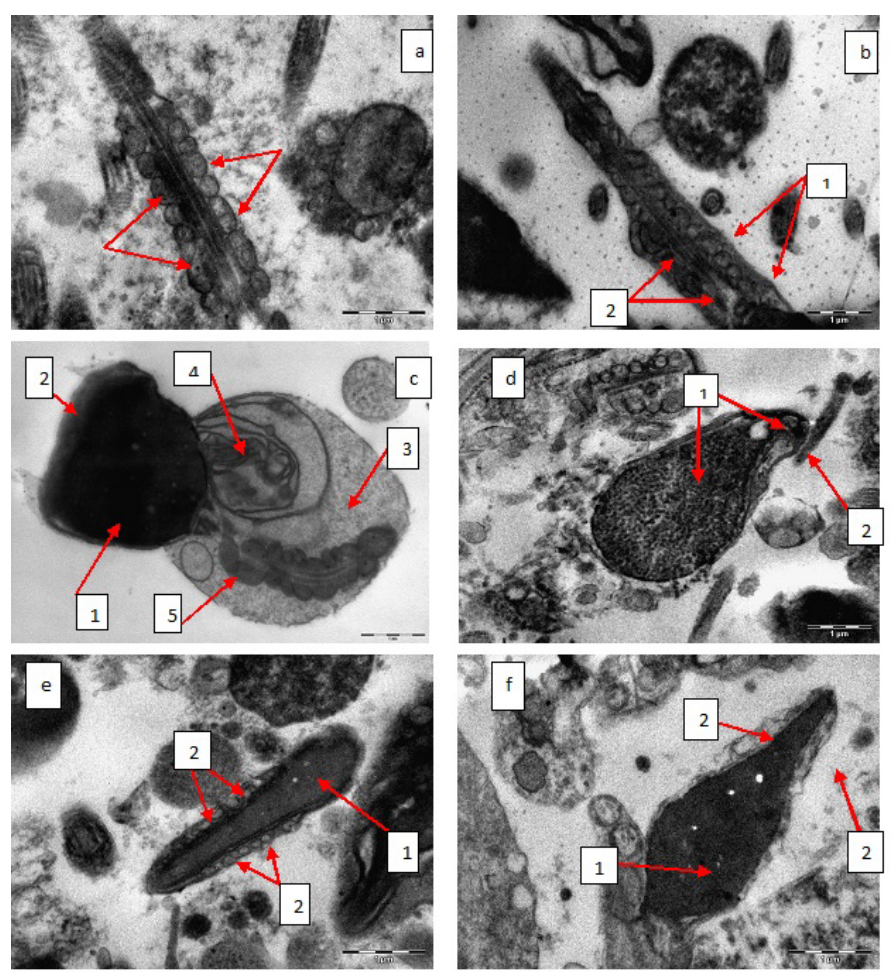

Figure 3. a - spermatozoa filament middle part, mitochondria swelling, cristas disrupture, clarification of mitochondrial matrix (is shown by arrows), spiral boxing around axoneme is intact, x 14000 ; b - spermatozoa filament middle part, mitochondria swelling, cristas disrupture, clarification of mitochondrial matrix (1), spiral boxing around axoneme is broken (2), x 14 000; c - spermatozoa dysplasia, abnormal head shape (1), acrosome degradation (2), cytoplasmic droplet in the area of spermatozoa filament middle part (3), hyperplastic nuclear membrane (4), mitochondria spiral boxing is broken (5), x 11000 ; d spermatozoa head, granular vacuolated chromatin (1), acrosome swelling and vacuolization (2), x 14 000; e - spermatozoa head, mature homogenous chromatin (1), acrosome swelling and vacuolization (2), x 11000 ; f - spermatozoa head, mature chromatin (1), acrosome degradation (2), x 14000 .

in the process of spermatogenesis damaging impact on the nuclear membrane occurs, which results in changes in physical and chemical properties of the membrane, its flowability, and changes in membrane potential. With spermatozoon maturation chromatin compaction occurs, which combined with changing properties of the membrane leads to its redundancy. In the process of spermatogenesis cytoplasm part in the form of residual body is disconnected from the spermatozoon and is phagocytosed by the Sertoli cells. Part of cytoplasm with mitochondria and areas of redundant nuclear membrane is shifted in caudal direction and then covers the entire spermatozoon neck, or in the form of large cytoplasmic droplet is localized in the bottom area of spermatozoon head, the middle part of the filament, thus significantly limiting its motility.

In addition, it was identified that patients with asthenozoospermia had pathological changes of chromatin (granularity, vacuolization) and acrosome (swelling, vacuolization, premature degradation) in spermatozoa heads.

As it is known from data presented in the cited literature, the influence of disordering factors on mature spermatozoa generally results in mitochondria pathological change. It is confirmed by the results obtained in our study. Thus each case requires individual approach in diagnosis identification, which should be based not only on such parameters as quantity, motility and morphology of spermatozoa, but also the level of oxidative and antioxidant status and functional tests must be taken into account, which provides data for comprehensive evaluation of spermatozoa fertilizing potential of a particular person.

Thus, we can assume that mitochondria pathological changes, nuclear membrane hyperplasia, identified using electron microscopic spermatozoa study are the most frequent and influential causes of asthenozoospermia.

\section{Conclusion}

1. There are no ultrastructural changes in the most number of cells within normal spermatozoa motility.

2. When there is spermatozoa motility disorder the number of spermatozoa with mitochondria changes increases as well as the number of spermatozoa with cytoplasmic droplet in the middle part of the filament that contains filament with signs of dysplasia, hyperplastic nuclear membrane, increases.

3. Mitochondria are the least stable structures of spermatozoa filament; other structures are less exposed to pathological changes.

4. If asthenozoospermia is identified the number of spermatozoa with chromatin and acrosome pathological changes increases.

Asthenozoospermia is the most common spermatozoa pathobiology and even a slight lowering of spermatozoa motility may be considered as subtle and early indicator of ejaculate quality deterioration.

\section{References}

1. Comhaire F, Vermeulen L (1995) Human semen analysis. Hum Reprod Update 1: 343362. [Crossref]

2. Evdokimov EE, Semenov TO (2006) Violation of spermatogenesis in varicocele Pathogenesis and prognosis of treatment. Urology and Genital Surgery 3: 12-18.

3. Jarow JP, Coburn M, Sigman M (1996) Incidence of varicoceles in men with primary and secondary infertility. Urology 47: 73-76. [Crossref]

4. Buffone MG, Brugo-Olmedo S, Calamera JC, Verstraeten SV, Urrutia F, et al. (2006) Decreased protein tyrosine phosphorylation and membrane fluidity in spermatozoa from infertile men with varicocele. Mol Reprod Dev 73: 1591-1599. [Crossref]

5. Agarwal A, Prabakaran S, Allamaneni SS (2006) Relationship between oxidative stress, varicocele and infertility: a meta-analysis. Reprod Biomed Online 12: 630-633. [Crossref]

6. Soufir JC (1985) [Azoospermia, asthenozoospermia and seminal biochemistry]. Ann Biol Clin (Paris) 43: 67-70. [Crossref]

7. Omu AE, Dashti H, Al-Othman S (1998) Treatment of asthenozoospermia with zinc sulphate: andrological, immunological and obstetric outcome. Eur J Obstet Gynecol Reprod Biol 79: 179-84.

8. Fraczek M, Kurpisz M (2007) Inflammatory mediators exert toxic effects of oxidative stress on human spermatozoa. J Androl 28: 325-333. [Crossref]

9. Kortebani G, Gonzales GF, Barrera C, Mazzolli AB (1992) Leucocyte populations in semen and male accessory gland function: relationship with antisperm antibodies and seminal quality. Andrologia 24: 197-204. [Crossref]

10. Kullisaar T, Türk S, Punab M, Korrovits P, Kisand K, et al. (2008) Oxidative stress in leucocytospermic prostatitis patients: preliminary results. Andrologia 40: 161-172. [Crossref]

11. Ahlgren G, Rannevik G, Lilja H (1995) Impaired secretory function of the prostate in men with oligo-asthenozoospermia. J Androl 16: 491-498. [Crossref]

12. Madar J, Urbánek V, Chaloupková A, Nouza K, Kinský R (2002) Role of sperm antibodies and cellular autoimmunity to sperm in the pathogenesis of male infertility. Ceska Gynekol 67: 3-7. [Crossref]

13. Nazarov SB, Kuzmenko GN, O-Gi-Ho EA, Sitnikova OG, Popova IG, et al. (2010) 
Reproductive problems 2: 60-62.

14. Trummer H, Ramschak-Schwarzer S, Haas J, Habermann H, Pummer K, et al. (2001) Thyroid hormones and thyroid antibodies in infertile males. Fertil Steril 76: 254-257. [Crossref]

15. Sahoo DK, Roy A, Bhanja S, Chainy GB (2008) Hypothyroidism impairs antioxidant defence system and testicular physiology during development and maturation. Gen Comp Endocrinol 156: 63-70.

16. Merino G, Carranza-Lira S, Martinez-Chéquer JC, Barahona E, Morán C, et al. (1997) Hyperprolactinemia in men with asthenozoospermia, oligozoospermia, or azoospermia. Arch Androl 38: 201-206. [Crossref]

17. Check JH, Lurie D, Vetter BH (1995) Sera gonadotropins, testosterone, and prolactin levels in men with oligozoospermia or asthenozoospermia. Arch Androl 35: 57-61. [Crossref]

18. Singer R, Ben-Bassat M, Malik Z, Sagiv M, Ravid A, et al. (1986) Oligozoospermia, asthenozoospermia, and sperm abnormalities in ex-addict to heroin, morphine, and hashish. Arch Androl 16: 167-174. [Crossref]

19. Zini A, Phillips S, Courchesne A, Boman JM, Baazeem A, et al. (2010) Alcohol intake and cigarette smoking: Impact of two major lifestyle factors on male fertility. Pathology and Microbiology 53: 35-40.

20. Gaur DS, Talekar M, Pathak VP (2007) Effect of cigarette smoking on semen quality of infertile men. Singapore Med J 48: 119-123. [Crossref]

21. Mostafa T, Tawadrous G, Roaia MM, Amer MK, Kader RA, et al. (2006) Effect of smoking on seminal plasma ascorbic acid in infertile and fertile males. Andrologia 38 : 221-224. [Crossref]
22. Viloria T, Meseguer M, Martínez-Conejero JA, O’Connor JE, Remohí J, et al. (2010) Cigarette smoking affects specific sperm oxidative defenses but does not cause oxidative DNA damage in infertile men. Fertil Steril 94: 631-637.

23. Dolgov VV, Lugovskaya SA, Mironov II (2006) Laboratory diagnosis of male infertility 6: 92-93.

24. Haidl G (1994) Asthenozoospermia-a multifactorial symptom. Fortschr Med 112: 489 491. [Crossref]

25. Mobberley MA (2010) Electron microscopy in the investigation of asthenozoospermia Br J Biomed Sci 67: 92-100. [Crossref]

26. Shamsi MB, Kumar R, Bhatt A, Bamezai RN, Kumar R, et al. (2008) Mitochondria DNA Mutations in etiopathogenesis of male infertility. Indian $J$ Urol 24: 150-154. [Crossref]

27. Venkatesh S, Deecaraman M, Kumar R, Shamsi MB, Dada R (2009) Role of reactive oxygen species in the pathogenesis of mitochondrial DNA (mtDNA) mutations in male infertility. Indian J Med Res 129: 127-137. [Crossref]

28. Wang X, Sharma RK, Gupta A, George V, Thomas AJ, et al. (2003) Alterations in mitochondria membrane potential and oxidative stress in infertile men: a psrospective observational study. Fertil Steril 80 Suppl 2: 844-850. [Crossref]

29. Benoff S, Auborn K, Marmar JL, Hurley IR (2009) Role of reactive oxygen species in the pathogenesis of mitochondrial DNA (mtDNA) mutations in male infertility. $I J$ Med Res 129: 127-137.

30. Sun ZM, Ding CF, Yan ZZ, Bao YZ (2007) Ultrastructure and function of mitochondria in idiopathic asthenospermia: study of 151 cases. Zhonghua Yi Xue Za Zhi 87: 12631265 .

Copyright: (2016 Pichugova Svetlana V. This is an open-access article distributed under the terms of the Creative Commons Attribution License, which permits unrestricted use, distribution, and reproduction in any medium, provided the original author and source are credited. 